\title{
Using a personal digital assistant enhances gather- ing of patient data on an acute pain management service: a pilot study
}

\author{
[L'utilisation d'un assistant numérique personnel facilite la cueillette de données \\ d'un service de traitement de la douleur aiguë : une étude pilote]
}

Elizabeth G. VanDenKerkhof RN DRPH, David H. Goldstein MB BCH MSc FRCPC, Jeremy Lane MD, Michael J. Rimmer, Janice P. Van Dijk RN MHSC

\begin{abstract}
Purpose: Handheld computer technology provides a unique opportunity for health care professionals to access real time or near real time patient information and evidence-based resources at the point-of-care. The purpose of this study was to assess one physician's experience using acute pain assessment software on a personal digital assistant (PDA) to assess patients on an acute pain management service (APMS).
\end{abstract}

Methods: Using a historical control and a "time and motion" study design, comparisons were made on acute pain assessment time and comprehensiveness when patient assessments are documented on a PDA vs the current paper-based method.

Results: The study physician (a PDA-user) reported feeling comfortable with the assessment software after five patient assessments. PDA assessments were more likely to contain documentation regarding pain and side effects (e.g., nausea, pruritus, hypotension) than the paper assessments. The median time of the "assessment only" component of the patient encounter was $53 \mathrm{sec}$ longer using the PDA compared to paper $(P<0.00)$, however, the median "total encounter" (chart review, assessment, documentation) time was $74 \mathrm{sec}$ shorter using the PDA vs paper $(P<0.00)$.

Discussion: The findings of this preliminary study suggest that the PDA is a reliable tool that meets the data management requirements within an APMS setting. This study found that patient assessments documented using acute pain software developed for use on a PDA were as efficient and content-rich as paper assessments. The PDA may even enhance the efficiency of the patient assessment process through the provision of more comprehensive digital data for research, clinical, and administrative needs.
Objectif : L'ordinateur nomade offre une chance unique aux professionnels de la santé d'accéder en temps réel ou presque à des renseignements sur le patient et à des ressources cliniques sur place. Notre objectif était d'estimer l'expérience d'un médecin qui utilise le logiciel d'évaluation de la douleur aiguë sur un assistant numérique personnel (ANP) pour examiner les patients d'un service de traitement de la douleur aiguë (STDA).

Méthode : Dans notre étude de type contrôle historique et tempsmouvement, nous avons comparé le temps et la représentativité de l'évaluation de la douleur aiguë pour des études de patients documentées sur ANP vs la méthode courante sur papier.

Résultats : Le médecin (un utilisateur d'ANP) s'est dit à l'aise avec le logiciel après cinq évaluations. Les examens avec l'ANP ont plus de chance de contenir de la documentation sur la douleur et les effets secondaires (comme les nausées, le prurit, l'hypotension) que celles sur papier. Le temps moyen de la partie «évaluation seule» de la rencontre avec le patient a été plus longue de $53 \mathrm{sec}$ avec l'ANP, comparé au papier $(P<0,00)$, mais le temps moyen de «l'entrevue complète» (revue du dossier, évaluation, documentation) a été plus court de 74 sec avec l'ANP vs le papier $(P<0,00)$.

Discussion : Les résultats de cette étude préliminaire indiquent que l'ANP est un outil fiable qui répond aux exigences de gestion des données dans le cadre d'un STDA. Les évaluations de patients documentées à l'aide du logiciel sur la douleur aiguë, mis au point pour un usage sur ANP, ont été aussi efficaces et riches de contenu que les évaluations sur papier. L'ANP peut même améliorer le procédé d'évaluation du patient en fournissant plus de données numériques détaillées pour la recherche, les besoins cliniques et administratifs.

From the Department of Anesthesiology, Kingston General Hospital, Queen's University, Kingston, Ontario, Canada.

Address correspondence to: Dr. Elizabeth G. VanDenKerkhof, Department of Anesthesiology, Kingston General Hospital, 76 Stuart St., Kingston, Ontario K7L 2V7, Canada. Phone: 613-549-6666, ext. 3964; Fax: 613-548-1375; Email: ev5@post.queensu.ca

Financial support: This paper is part of the research supported by grants from the Canadian Foundation for Innovation, the Ontario Innovation Trust, Queen's University and Kingston General Hospital. Financial and/or in kind support has also been provided by the Claire Nelson Bequest Fund, Bickell Foundation, Health Evidence Application and Linkage Network, Purdue- Pharma, Cisco, Compaq and Smith Industries.

Accepted for publication September 30, 2002.

Revision accepted January 8, 2003. 


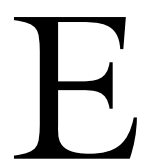

LECTRONIC patient records currently exist primarily in the form of the hospital information systems (HIS) with a desktop computer as the user interface. To a lesser extent, point-of-care tools such as personal digital assistants (PDAs) are being used by physicians, primarily to access clinical practice guidelines and alerts. ${ }^{1-5}$ PDAs at the point-of-care have been shown to provide accurate, timely and concise information for clinical audit and data collection, ${ }^{6-9}$ and when compared to paper they produced fewer errors and took less time to process. ${ }^{8,10}$ The few studies published in the nursing literature on the use of point-of-care tools for nursing documentation suggest that pointof-care tools may improve nursing documentation. ${ }^{11,12}$ The authors are not aware of studies in the literature assessing the use of point-of-care tools by physicians for documentation of patient assessments. The purpose of this study was to assess the use of point-of-care technology for documentation of patient assessments on an acute pain management service (APMS). A comparison was made between time and comprehensiveness of the acute pain assessment encounter using a PDA vs the current paper-based method (PM) of documentation.

\section{Methods}

This pilot study was designed to provide a simple comparison between traditional paper-based assessments and electronic assessments with respect to assessment content and time, and at the same time test, in a clinical setting, acute pain assessment software developed for use on a PDA at the point-of-care. We proposed that the patient assessment content would be the same for both the traditional PM and the PDA method of documentation, and the length of time to complete an acute pain patient assessment would not vary between the PM and the PDA documentation.

Approval to complete this study was given by the Queen's University Health Sciences Research Ethics Board. Patient assessments were performed in the routine manner and the study physician did not alter patient care provided by the APMS. A thorough review was performed of the current flow of patients through the APMS at Kingston General Hospital, followed by an adaptation of the flow to include electronic assessments. ${ }^{13}$ Based on these findings, plus the findings of our national survey of academic acute pain services, ${ }^{14}$ a portable assessment record was developed using PDAs on the Palm Operating System (Palm Computing® Platform, Palm Inc., CA, USA).

A before/after study was designed using historical controls to compare the PM assessments with the
PDA assessments. This approach was selected over a randomized parallel design in order to blind the study physician to the contents of the APMS software. No studies were identified in the literature that assessed the impact on patient care of the use of PDAs to document patient assessments (i.e., do physicians document differently when using a PDA?). Given that clinicians may alter their documentation patterns, it was believed that this pilot study should be carried out separate from regular patient rounds. All of the information that was available during APMS rounds was made available to the study physician during both the PM and the PDA assessments; however, the data collected by the study physician were only made available for study purposes and did not become part of the official patient chart. One physician (third year anesthesiology resident) who had spent one month with the APMS as part of his training was recruited to test the tool. The rationale for having only one physician participate in the study was that this was the first test of the PDA with the assessment software in the clinical setting, therefore we believed it was prudent to test it outside of patient rounds before introducing it into patient rounds in a study involving the APMS physicians. The alternate solution was to have a research assistant carry out the study, however, it was believed that this would have resulted in an even less accurate depiction of the point-of-care technology as a clinical assessment tool.

The study was conducted over a three-week period in February/March 2001. The control group (PM) was recruited in the first half of the study period (February 19-27, 2001) and the treatment group (PDA) was recruited in the second half of the study period (February 29-March 9, 2001). For both groups the study physician spent the initiation period (two days) becoming familiar with the data collection process and the timing of assessments. Any issues that arose during that time were addressed before the formal data collection process began. After the initiation period for each arm, five days were spent recruiting patients and recording assessments. For the PM arm the study physician had access to the complete patient chart but documented on a blank chart that included a doctor's order sheet and a progress note sheet. The study physician was asked to record his assessment and orders as if he were performing and recording a routine APMS assessment. The APMS software was not downloaded to the study physician's PDA until completion of the PM arm of the study. The study physician spent the first two days of the PDA arm of the study becoming familiar with the software followed by five days of data collection. After all study assessments were completed, the 
PM assessments were entered into an Excel spreadsheet (Appendix available at www.cja-jca.org). In both the PM and the PDA arms, free text not captured by the APMS variables, was captured under the open-ended variable called "Doctors Notes". Patient confidentiality on the PDA was addressed through the use of a login identification number and a password which the study physician was required to enter before patient data populated the PDA screen.

The APMS software included standard pain scoring systems (e.g., numeric rating scale), as well as an extensive list of potential drug-related side effects (Appendix available at www.cja-jca.org). Items could be selected by checking tick boxes or items from a drop-down menu. The study physician was not forced to follow a specific order, but could move freely between screens without having to make a selection. A depiction of a standard screen can be found in Figure 1. The study physician was asked to record the findings of the assessment on the PDA, either in front of the patient or outside the patient's room.

A consistent timing mechanism was used for both arms of the study. The Queen's University anesthesia informatics laboratory (QUAIL) developed a stopwatch program for use on a Palm PDA. The program variables included the patient's name, unique clinical record (CR) number, study arm, and the five assessment time points outlined below. The study physician carried a second PDA with the stopwatch software. At the beginning of each day the PDA timer was synchronized with a timing database on the APMS server. The patient's name, CR number and study arm for all study patients were transferred from the timing database on the APMS server to the PDA timer. The study physician selected the patient on the PDA from a drop-down menu. Time was recorded by tapping the screen as he progressed through each stage of the patient encounter. The timer was set up to calculate the time interval for each encounter. At the end of each day the PDA was synchronized with the timing database on the APMS server. The timing of the assessments began outside of the patient's room while reviewing the patient's chart, and ended when all documentation was complete. The five time points were as follows: 1) outside of the patient's room and about to begin the chart review; 2) entering the patient's room (after reviewing patient's chart); 3) begin the patient assessment (after greeting patient); 4) end of assessment; and 5) recording completed. For the purpose of this study the time periods of interest were the assessment itself (time point 3 to time point 4 ), recording of the assessment (time point 4 to time point 5 ), and the total encounter time [chart review (time point 1 ) to recording completed (time point 5)].
Patients recruitment was standardized for both the PM and PDA arms of the study. The majority of patients are routinely admitted to the APMS by a consultation process initiated in the operating room between the surgeon and the anesthesiologist. At this time an APMS consult request is completed and the patient is entered onto the APMS list by the anesthesiologist. Alternately the patient may be entered onto the APMS list in the postanesthesia care unit by either the anesthesiologist or APMS nurse. This process flags the patient's record on the HIS indicating that the patient has been admitted to the APMS. Each morning the APMS nurse or anesthesiologist generates a paper list of patients on service from the HIS. For the purpose of this study, patients on the PM arm were identified for the study from this paper list generated from the HIS. The same mechanism was used for the PDA arm, except rather than print a paper list, an interface was developed between the HIS and the APMS server. Patient's names were transferred directly from the HIS to the APMS server and then downloaded via synchronization to the PDA.

PM and PDA assessment content and time were compared. The frequency of documentation of pain and side effect variables for the two methods were determined. Comparisons were made between the median and mean time (in seconds) required to perform the patient assessment, record the assessment, and the total encounter time. Tests for normality were performed using the Kolmogorov-Smirnov test. The data were found to be not normally distributed, hence, the Mann-Whitney test was used to test for differences in the median assessment, recording, and total encounter time for PDA vs paper. Repeated measures analysis was not used, as the relationship between the various components of the total patient encounter time was not relevant to this analysis. In order to account for dependency between outcomes, the analysis was performed twice, on all patient assessments and on the first assessment only for each patient. Results were similar on data including only the first assessment per patient and on data including all assessments per patient; therefore, all assessments per patient were included and treated independently in the final analysis. Sample size was calculated using an alpha of 0.05 and a power of $80 \%$ with an effect size of $60 \mathrm{sec}$ and standard deviation of $84 \mathrm{sec}$. Based on these requirements, it was determined that at least 32 patients would be required per arm. The goal was to perform 100 PM assessments and 100 PDA assessments.

\section{Results}

All patients who were approached agreed to participate in the study. The study physician performed 
patient rounds twice a day. He was encouraged to perform the assessments in his usual manner. For him this meant that charting on the PM arm took place outside the patient's room in the progress notes and the physician's order sheet, and charting on the PDA took place in front of the patient during the patient assessment. The study physician, who was familiar with PDAs prior to the study, reported feeling comfortable with the software after approximately four to five patient assessments and within a few hours on the first day of the PDA arm initiation period. One hundred assessments were performed on 44 patients in the PM arm and 104 assessments on 40 patients were completed on the PDA. Data integrity for the first ten PDA assessments was called into question due to a synchronization problem during the first download of data from the PDA to the APMS server. Hence, in order to ensure data integrity the first ten PDA assessments were excluded from the database. The final sample size on the PDA arm, for purposes of the analysis, was 94 valid assessments on 30 patients.

The age and sex characteristics did not differ between the two arms. There were slightly more males than females in both arms of the study and the average age in each arm was approximately $60 \mathrm{yr}$. Table I lists the percent of times the ten most frequently documented side effects were included in the paper record, and the corresponding percent of times the side effects were documented on the PDA. All ten side effects were recorded for virtually all the PDA assessments, while on the PM arm the percent of times the side effects were recorded ranged from $100 \%$ for hypotension down to $5 \%$ for headache. No significant difference was found between documentation of the numeric rating scale (NRS) score for paper $p s$ PDA (Table II), however, the nature of pain was much more likely to be described on the PDA vs PM arm. Aspects of the patient assessment times are reported in Table III. Assessment time only, recording time only, assessment plus recording time, and total patient encounter time are reported. There were statistically significant differences between the PDA and paper arms in median assessment time for all time-points in the assessment process. The median time to complete the assessment only was $\mathbf{5 3} \mathrm{sec}$ longer for the PDA arm compared to paper arm $(P=0.00)$, however, when recording was included, the median time was $61 \mathrm{sec}$ shorter in the PDA arm compared to the paper arm $(P=0.00)$. The median PDA time remained significantly shorter than the median paper time when the total encounter was considered $(227$ vs $301 \mathrm{sec}, P=$ $0.00)$. Figure 2 depicts the median total encounter time by day of data collection for each arm when all patient assessments were included in the analysis and when only
TABLE I Frequency of documentation of the ten most frequently collected side effects variables during acute pain assessments using the current paper-based method of charting vs using a PDA for charting

\begin{tabular}{lll}
\hline Variables & $\begin{array}{l}\text { Paper }(n=100) \\
n(\%)\end{array}$ & $\begin{array}{l}\text { PDA }(n=94) \\
n(\%)\end{array}$ \\
\hline Hypotension & $100(100)$ & $93(99)$ \\
Pruritus score & $98(98)$ & $94(100)$ \\
Nausea & $93(93)$ & $94(100)$ \\
Vomiting & $81(81)$ & $94(100)$ \\
Effectiveness of therapy & $12(12)$ & $93(99)$ \\
NPO status & $8(8)$ & $94(100)$ \\
Ambulation & $7(7)$ & $92(98)$ \\
Level of sedation & $7(7)$ & $94(100)$ \\
Insomnia & $6(6)$ & $94(100)$ \\
Headache & $5(5)$ & $93(99)$ \\
\hline
\end{tabular}

$\mathrm{PDA}=$ personnal digital assistant $\mathrm{NPO}=$ nil per os.

TABLE II Frequency of documentation of pain variables collected during acute pain assessments using the current paper-based method of charting $v s$ using a PDA for charting

\begin{tabular}{lll}
\hline & Paper & PDA \\
$n(\%)(n=100)$ & $n(\%)(n=94)$ \\
NRS score & $97(97)$ & $94(100)$ \\
$\quad$ Rest & $90(90)$ & $94(100)$ \\
$\quad$ Active & $2(2)$ & \\
Free text documentation & $3(0)$ \\
$\quad$ Pain description & $n(3)$ & $12(13)$ \\
PCA/epidural comments & $n(\%)(n=67)$ & $n(\%)(n=51)$ \\
NRS Score at rest $>0$ & $2(3)$ & $50(98)$ \\
$\quad$ Characterization of pain & $0(0)$ & $48(94)$ \\
Location of pain & $0(0)$ & $29(57)$ \\
Duration of pain & $n(\%)(n=81)$ & $n(\%)(n=82)$ \\
NRS score active $>0$ & $1(1)$ & $93(99)$ \\
Characterization of pain & $1(1)$ & $90(96)$ \\
Location of pain & $0(0)$ & $41(50)$ \\
Duration of pain &
\end{tabular}

PDA = personnal digital assistant PCA $=$ patient controlled anal gesia; $\mathrm{NRS}=$ numeric rating scale.

TABLE III Median (interquartile range) time (in seconds) to completion of various aspects of the acute pain assessment using the current paper-based method $v s$ using a PDA

\begin{tabular}{|c|c|c|c|}
\hline Time points & $\begin{array}{l}\text { Paper }(n=100) \\
\text { Median }(I Q R+)\end{array}$ & $\begin{array}{l}\text { PDA }(n=94) \\
\text { Median }(I Q R+)\end{array}$ & Pvalue* \\
\hline $\begin{array}{l}\text { Review chart \& } \\
\text { greet patient }\end{array}$ & $29(19)$ & $16(9)$ & 0.000 \\
\hline Assessment only & $153(85)$ & $206(70)$ & 0.000 \\
\hline Record only & $119(40)$ & $0(1.3)$ & 0.000 \\
\hline Assessment \& recording & $269(103)$ & $208(70)$ & 0.000 \\
\hline Total time (seconds) & $301(92)$ & $227(71)$ & 0.000 \\
\hline
\end{tabular}

$\dagger$ Interquartile range; *Mann-Whitney test. PDA = personnal digital assistant. 


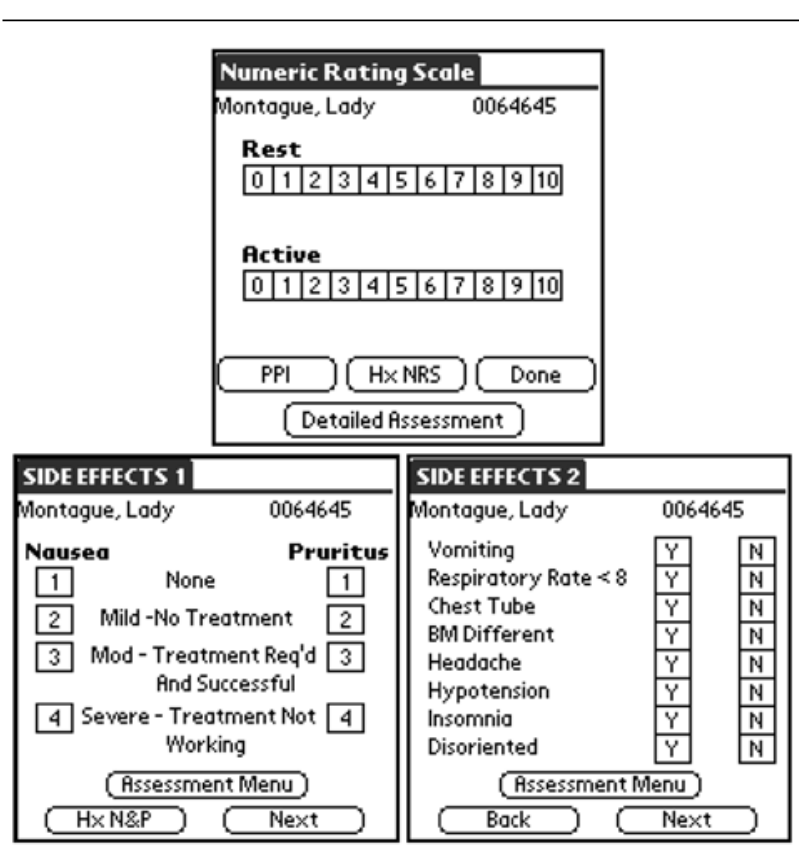

FIGURE 1 Standard screens of the personal digital assistant.

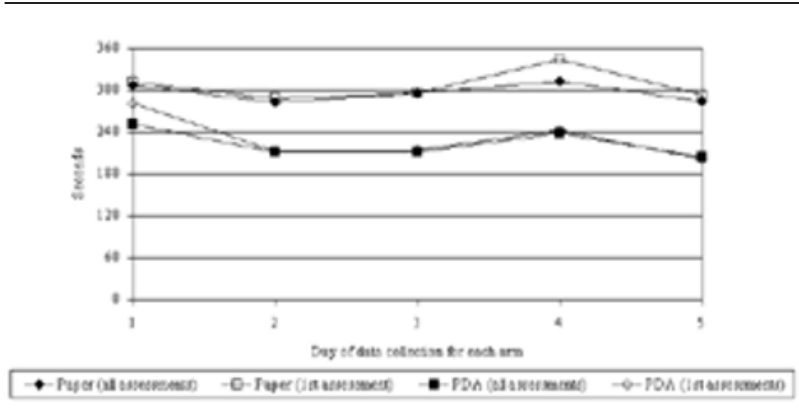

FIGURE 2 Median total time of patient encounter by day of assessment: paper $v s$ personal digital assistance.

the first assessment per patient was included. Encounter time was slightly higher on day one of the PDA arm; however, the difference in encounter time between the PDA and the PM was consistent over days two to five. Encounter times did not vary significantly between the first patient assessment and all patient assessments.

\section{Discussion}

In this study the time to completion and the content of acute pain assessments were compared for assess- ments documented using the current paper-based method $p s$ using a PDA at the point-of-care. The study was carried out outside of patient rounds by an anesthesiology resident with previous experience using PDAs and with experience performing acute pain patient assessments. The total patient encounter time was found to be significantly shorter (median, $301 \mathrm{vs}$ $227 \mathrm{sec}, P=0.00$ ) and the amount of information documented was greater on the PDA arm compared to the paper arm. These results are consistent with findings in the literature, where hand held electronic data capture was shown to be acceptable, reliable, efficient, and qualitatively as rich as paper and pencil methods. ${ }^{7}$ The decreased time spent on patient encounters on the PDA arm was a reflection of decreased time spent recording the assessment while the actual time spent with the patient was similar between the two groups. In this institution a savings of one minute per assessment reflects a 30-45 min savings per day for the APMS physician. This represents approximately $12.5 \%$ percent of the average time spent on APMS patient rounds per day. The availability of the assessment in electronic format may also lead to time saved during clinical audits and research endeavours, while decreasing data entry error rates.

In reviewing the discrepancy in content between the two arms, several factors must be addressed. Information may have been forgotten on the PM arm because recording took place outside of the patient's room. Lack of documentation on the PM arm may also be indicative of a negative result or a side effect thought to be irrelevant to the clinical situation. For example, if a patient does not have a headache the clinician may not record this information unless it is deemed to be relevant to the situation (e.g., epidural or spinal anesthetic). Therefore, without prompting by the physician, a side effect such as headache or pruritus may not be captured unless the patient offers the information. Patients are not necessarily versed in side effects associated with therapy (e.g., pruritus, headaches). Such symptoms may not be viewed by patients as being associated with pain and therefore may not be reported to the APMS. Prompts on the PDA may encourage physicians to ask patients about specific side effects associated with therapy, thereby resulting in a more comprehensive approach to patient care. In spite of the discrepancy in documentation content, the primary opioid-related side effects found in the literature ${ }^{14,15}$ - hypotension, pruritus and nausea - were also the most frequently documented side effects in both arms of the study. Finally, a paper check list may well have resulted in the same amount of data being collected in the PM arm, however, the purpose 
of this study was to compare the use of a PDA to the current "gold standard", which in most institutions is not in the form of a checklist, but rather a blank assessment record. A paper checklist would also not result in data that is readily available, with the capacity to be queried, for ongoing clinical, quality assurance, administrative and research purposes.

The use of the NRS to document level of pain was consistently reported in both arms of the study. The use of this measure to document pain levels is supported by the literature. ${ }^{14,16,17}$ In patients reporting a NRS score greater than 0 , the study physician elaborated on the characterization and location of the reported pain in the majority of assessments on the PDA arm, but not on the PM arm. The documentation of detailed pain information on the PDA arm was likely due to the prompts provided by the PDA. This impact on documentation content is an important finding, as the identification and characterization of pain is necessary in order to distinguish between expected surgical pain (visceral or somatic) and other potential postoperative complications (e.g., acute neuropathic pain).

The various aspects of the patient encounter presented in Table III indicate that while recording on the patient's chart occurred after the patient assessment in the PM, recording on the PDA occurred during the assessment. This may also account for the discrepancy in comprehensiveness of documentation between the two arms. Documentation on the PDA may also be influenced by physician practices. Practice patterns, including documentation, may differ for physicians-intraining compared to physicians with extensive experience. While the study physician used the PDA as a guide to his assessment, our early impression from experienced physicians is that they view electronic data capture as a data entry tool. This in combination with the tendency by many physicians to document on the patient's chart outside of the patient's room may result in experienced physicians documenting differently on the PDA than our findings suggest. In particular, when recording occurs after the assessment, outside of the patient's room, the assessment prompts provided on the PDA may not be fully utilized. Unlike the current paper record, the PDA may increase assessment documentation content to a certain extent by reminding physicians to document information they observed or obtained from the patient. However, prompts for data the physician did not collect are less likely to be utilized, as it would require that the physician return to the patient's room to obtain the information.

One might expect total patient encounter time to decrease as the study physician became facile in using the PDA assessment tool, however this did not occur as illustrated in Figure 2. The consistent nature of the difference in time between the two methods indicates that the historical nature of the study did not adversely affect the findings. The study physician had a history of PDA use, but he had no prior experience with the assessment software, therefore, as expected, the total encounter time was slightly longer on the first day of the PDA arm, however, on subsequent days the time remained relatively consistent.

The findings of this study suggest that the use of PDAs at the point-of-care is equivalent to paper and may even enhance the patient assessment process, while providing more comprehensive data for research, clini$\mathrm{cal}$, and administrative needs. The limited adoption of these real-time systems often occurs because of the lack of infrastructure required to support these systems in most hospitals. ${ }^{18}$ However, with the introduction of PDAs and wireless technology into the health care setting, a viable opportunity exists to bring real-time information to the patient's side, while collecting valuable patient information in an electronic format. ${ }^{3,4,12,19,20} \mathrm{~A}$ report by Harris Interactive indicates that approximately $26 \%$ of physicians now use handheld computers in their practice and they predict that this number may increase to $50 \%$ by $2005 .{ }^{21}$

Study strengths include the use of a single investigator with experience in both acute pain patient assessments and in the use of PDAs. While it could be argued that some of the differences between groups could be attributed to increased efficiency in performing patient assessments and in the use of the PDA, it is believed that the differences between groups are largely a reflection of real differences in the two methods. The study occurred over a very short period of time (three weeks); therefore issues such as changes in physician practices and the evolution of technology did not affect the findings. Due to the paucity of data in the literature assessing the impact of technology on health care and clinical decision-making at the bedside, it was deemed relevant to conduct the study outside of formal patient rounds. The study physician simulated patient rounds, but decisions he made regarding patient care were not carried out. In situations where he felt that the information he obtained should be acted upon, he notified the APMS physician. All other aspects of the patient encounter were consistent with routine APMS rounds. The study took place within a clinical setting and all of the data normally available to the APMS were available to the study physician. A further strength of the study is that a time and motion study was carried out in a consistent manner for both arms using a PDA with stopwatch software. 
Study limitations include the non-randomized nature of the study. However, it was felt that the disadvantage of not using a randomized design was outweighed by the advantage of the study physician not being influenced by the contents of the acute pain software when performing PM assessments. An additional limitation is that only one physician, who was early in his specialty training, participated in the study. As such, the results can only be generalized to physicians in similar circumstances. The study physician was a previous PDA owner. Non-PDA owners may perform differently with respect to both timing and comprehensiveness of data collection. The study physician found the tool to be very useful as a guide for the assessment. It is expected that experienced physicians are less likely to be impressed by the usefulness of the tool as a guide to their assessments. Assessments were performed after APMS rounds, resulting in potentially shorter than usual assessment times, however, this bias existed in both arms of the study. A further limitation to the study was the study physician was responsible for timing his own assessments, which may have affected timing. Again this limitation was present in both arms and any impact on timing was likely experienced in the PDA arm. The handling of two PDAs (the PDA timer and the assessment PDA) may have been awkward, resulting in increased assessment time on the PDA arm. A final limitation of the study was the loss of the first ten PDA patients during data synchronization. Due to over-recruitment of patients to the study (40 PDA patients), this loss resulted in a sample size of 30 rather than the 32 required to achieve a significant result with a $60 \mathrm{sec}$ difference between arms. Given that the difference in time between arms for the total patient encounter was greater than $60 \mathrm{sec}$ plus the fact that the final analysis included all patient assessments rather than only one assessment per patient, the study had ample power to detect a significant result.

The findings of this study suggest that the PDA is a reliable tool that meets the data management requirements within an APMS setting. Since the ultimate goal in developing point-of-care systems is to have access to near real-time information to assist in evidence-based clinical decision-making at the bedside, hand held technology linked with the HIS appears to be a viable option in an APMS setting. Research and development efforts play an important role in the adoption of these technologies in a clinical environment. ${ }^{22} \mathrm{~A}$ follow-up clinical trial has recently been completed comparing consultant anesthesiologist's assessment time and comprehensiveness using PDAs vs paper on APMS rounds. An additional study is underway looking at the feasibility of having patients complete their self-administered pre-admission questionnaire using a computerized interface. Future trials will investigate the effectiveness and practicality of the hand held in concert with wireless computer technologies in the APMS environment, and the implementation and integration of an electronic preoperative consultation record and an intraoperative automated record keeper.

\section{Acknowledgements}

We would like to thank the software development team - Lynn Douglas, Jeff Babcock, Mat Hartman, Darcy Dick, and Aaron Visser. We would also like to thank Dr. Ian Gilron for his thoughtful comments on this manuscript.

\section{References}

1 Turban E. Decision Support and Expert Systems: Management Support Systems, 4th ed. Englewood Cliffs, NJ: Prentice Hall; 1995.

2 Shortliffe EH. Computer-Based Medical Consultations: MYCIN. New York, NY: Elsevier/North; 1976.

3 Snyder-Halpern R. Assessing health care setting readiness for point of care computerized clinical decision support system innovations. Outcomes Manag Nurs Pract 1999; 3: 118-27.

4 Lapinsky SE, Weshler J, Mehta S, Varkul M, Hallett D, Stewart TE. Handheld computers in critical care. Crit Care 2001; 5: 227-31.

5 Ebell MH, Barry HC. InfoRetriever: rapid access to evidence-based information on a hand- held computer. MD Comput 1998; 15: 289, 292-7.

6 Wofford MM, Secan R, Herman C, Moran WP, Wofford JL. Clinical documentation: the handheld computer as a survival tool. MD Comput 1998; 15 : $352-4,356,358$.

7 Robinson D. Hand-held computers in clinical audit. Sr Nurse 1993; 13: 14-7.

8 Curl M, Robinson D. Hand-held computers in clinical audit: a comparison with established paper and pencil methods. Int J Health Care Qual Assur 1994; 7: 16-20.

9 Robinson D. Data capture using hand-held computers. J Psychiatr Ment Health Nurs 1994; 1: 126-7.

10 Lal SO, Smith FW, Davis JP, et al. Palm computer demonstrates a fast and accurate means of burn data collection. J Burn Care Rehabil 2000; 21: 559-61.

11 Nahm R, Poston I. Measurement of the effects of an integrated, point-of-care computer system on quality of nursing documentation and patient satisfaction. Comput Nurs 2000; 18: 220-9.

12 Young PM, Leung RM, Ho LM, McGhee SM. An evalua- 
tion of the use of hand-held computers for bedside nursing care. Int J Med Inf 2001; 62: 189-93.

13 Goldstein DH, VanDenKerkhof EG, Rimmer MJ. New media. Can J Anesth 2002; 49: 749-54.

14 Goldstein DH, VanDenKerkhof EG. Organization of acute pain services in Canadian teaching hospitals. Can J Anesth 2001; 48: 17A (abstract).

15 Canadian Pharmacists Association, Compendium of Pharmaceuticals and Specialties. 35 ed. Ottawa: Canadian Pharmacists Association; 2002.

16 Goldstein DH, VanDenKerkhof EG, Sherlock R, Sherlock $J$, Harper $S$. How an audit of epidural patients in a community hospital setting resulted in the development of a formal acute pain management service. Pain Res Manage 2001; 6: 16-20.

17 Rawal N. 10 years of acute pain services-achievements and challenges. Reg Anesth Pain Med 1999; 24: 68-73.

18 Drazen EL, Metzger JB, Schneider MK. Patient Care Information Systems: Successful Design and Implementation. New York, NY: Springer-Verlag; 1995.

19 Smith CM. Is wireless technology at hand for healthcare? J AHIMA 2001; 72: 22, 24.

20 Larkin $M$. Can handheld computers improve the quality of care? Lancet 2001; 358: 1438.

21 Taylor H, Leitman R. Physicians' use of handheld personal computing devices increases from $15 \%$ in 1999 to $26 \%$ in 2001. Health Care News 2001; 1: 1-4.

22 Sittig DF, Jimison HB, Hazlehurst BL, et al. Techniques for identifying the applicability of new information management technologies in the clinical setting: an example focusing on handheld computers. Proc AMIA Symp 2000; 804-8. 